\title{
Modes with variable mass as an alternative in AdS / QCD models with chiral symmetry breaking
}

\author{
Alfredo Vega and Ivan Schmidt \\ Departamento de Física y Centro Científico Tecnológico de Valparaíso, \\ Universidad Técnica Federico Santa María, Casilla 110-V, Valparaíso, Chile.
}

(Dated: April 20, 2022)

\begin{abstract}
We consider a new possibility of incorporating chiral symmetry breaking in soft wall models with a quadratic dilaton and AdS metric. In particular, we allow the mass of the scalar modes propagating in the bulk to have a dependence on the holographical coordinate. In one of the models that we discuss, it is possible obtain a good meson spectra considering current quark masses, and in the other two models considered, good results can be obtained at the expense of considering rather large quark masses.

PACS numbers: 11.25.Tq, 11.25.Wx, 11.30.Qc

Keywords: holographical model, chiral symmetry breaking, AdS / QCD
\end{abstract}

\section{INTRODUCTION}

Within the range of hadronic properties that can be studied using AdS / QCD, we find models that incorporate effects of chiral symmetry breaking explicitly in the lagrangian [1, 2]. One kind of such models, known as hard wall, allows to break chiral symmetry both spontaneously and explicitly in an independent way, but the hadronic spectra calculated in this case turns out to be not good. This situation can be improved by the introduction a dilaton field, which in many articles is considered quadratic in the holographical coordinate z 3]. The obtained spectra has Regge behavior, but unfortunately it is not possible now to break chiral symmetry explicitly and spontaneously $[\underline{3}, 4]$.

The satisfactory implementation of chiral symmetry breaking, without sacrificing the hadronic spectra, is a problem that has attracted much interest lately. Examples of these kind of efforts can be found in 5-9]. Here we show a different alternative, since we consider that the mass for modes propagating inside the bulk can present a dependence on the holographical coordinate $z$, which could be due to the fact that operators associated to these modes might have an anomalous dimension $10-13$.

It is possible to find references to $\mathrm{z}$ dependent masses in the literature [10 13], where the authors suggest that the anomalous dimension of operators can be translated into z dependent masses for dual modes of these operators. This idea was used successfully in 13], where an holographic model without explicit chiral symmetry breaking was considered, and which can reproduce the hadronic spectrum for spin $1 / 2$ and $3 / 2$ baryons with with an arbitrary number of constituents. As is known for spin $1 / 2$ case, a dilaton field can not improve hard wall models, because this field is factorized from the equation that gives us the spectra [13, 14]. Other work related to mass varying in the bulk can be found in papers such as [15 18], where the mass changes in order to differentiate between the pion and other scalar mesons, or in AdS / QCD models that consider chiral symmetry breaking, for example in [6], where a vev is coupled to some modes, producing an effective $\mathrm{z}$ depending mass in the equation associated to some mesons.

In this article we consider an AdS / QCD model that takes into account effects of chiral symmetry breaking, with $\mathrm{z}$ dependent scalar mode masses. Notice that these modes are dual to the $q \bar{q}$ operator, which is not a conserved charge, and therefore this operator has an anomalous dimension. We consider three models called models I, II and III, each one related to the behavior of the vev when $z \rightarrow \infty$. For certain set of parameter we obtain that the lightest scalar meson has a mass lower that of the pion, contradicting well established properties of QCD [19, 20]. Nevertheless, this problem is not present for all cases, in fact model II allows us to get good results for the spectra with current quark masses, other models considered here needs quark masses with unreasonable large value. Therefore, the model II can be considered as a serious complementary alternative, different to the effort developed in [6 8], where the authors try to improve soft wall models by deforming the dilaton and/or the metrics.

The work consists of the following parts. Section II is a brief description of the model, where we write down the equations that describe the vev and the scalar, vector and axial vector mesons in the AdS side. In III we obtain a variable mass for the scalar modes. In section IV we discuss how to fix the parameters involved in this model, in order to obtain in section $\mathrm{V}$ the spectra with the parameters of the previous section. Finally, section VI is dedicated to expose the conclusions of this work.

\section{MODEL}

We consider the most usual version of soft wall AdS / QCD models, with the notation used in [6], which takes into account an $5 \mathrm{~d}$ AdS background defined by

$$
d s^{2}=\frac{R^{2}}{z^{2}}\left(\eta_{\mu \nu} d x^{\mu} d x^{\nu}+d z^{2}\right)
$$


TABLE I: Field content and dictionary of the model.

\begin{tabular}{c|c|c|c|c}
\hline \hline $4 \mathrm{D}: O(\mathrm{x})$ & $5 \mathrm{D}: \Phi(x, z)$ & $\mathrm{p}$ & $\Delta$ & $m_{5}^{2} R^{2}$ \\
\hline $\bar{q}_{L} \gamma^{\mu} t^{a} q_{L}$ & $A_{L \mu}^{a}$ & 1 & 3 & 0 \\
$\bar{q}_{R} \gamma^{\mu} t^{a} q_{R}$ & $A_{R \mu}^{a}$ & 1 & 3 & 0 \\
$\bar{q}_{R}^{\alpha} q_{L}^{\beta}$ & $\frac{1}{z} X$ & 0 & $3+\delta$ & $m_{5}^{2}(z) R^{2}$ \\
\hline \hline
\end{tabular}

where $\mathrm{R}$ is the AdS radius, the Minkowsky metric is $\eta_{\mu \nu}=\operatorname{diag}(-1,+1,+1,+1)$ and $\mathrm{z}$ is a holographical coordinate defined in $0 \leq z<\infty$. In this paper we consider a usual quadratic dilaton

$$
\phi(z)=\lambda^{2} z^{2}
$$

To describe chiral symmetry breaking in the mesonic sector in the $5 \mathrm{~d}$ AdS side, the action considers $S U(2)_{L} \times$ $S U(2)_{R}$ gauge fields and a scalar field $\mathrm{X}$. Such action is given by

$$
\begin{gathered}
S_{5}=-\int d^{5} x \sqrt{-g} e^{-\phi(z)} \operatorname{Tr}\left[|D X|^{2}+m_{X}^{2}(z)|X|^{2}\right. \\
\left.+\frac{1}{4 g_{5}^{2}}\left(F_{L}^{2}+F_{R}^{2}\right)\right]
\end{gathered}
$$

This action shows explicitly that the scalar modes masses are $\mathrm{z}$ dependent, which is the feature that distinguishes this model from other AdS / QCD models with chiral symmetry breaking.

Here $g_{5}^{2}=\frac{12 \pi^{2}}{N_{c}}$, where $N_{c}$ is the number of colors, and the fields $F_{L, R}$ are defined by

$$
F_{L, R}^{M N}=\partial^{M} A_{L, R}^{N}-\partial^{N} A_{L, R}^{M}-i\left[A_{L, R}^{M}, A_{L, R}^{N}\right]
$$

here $A_{L, R}^{M N}=A_{L, R}^{M N} t^{a}, \operatorname{Tr}\left[t^{a} t^{b}\right]=\frac{1}{2} \delta^{a b}$, and the covariant derivative is

$$
D^{M} X=\partial^{M} X-i A_{L}^{M} X+i X A_{R}^{M}
$$

The scalar field $\mathrm{X}$, which is dual to the operator $q \bar{q}$, has a vev given by

$$
X_{0}=\frac{v(z)}{2}
$$

which produces chiral symmetry breaking.

In Table I the fields included in model are shown and also his relationship with modes propagating in the bulk, according to AdS / CFT dictionary. Notice that the operator $q \bar{q}$ has an anomalous dimension $(\delta)$, which in turn produces a mass that depends on $\mathrm{z}$ for the modes dual to this operator, in agreement with [10, 13].

Starting from (3), the equations that describe the vev and the scalar, vector and axial vector mesons are

$$
\begin{gathered}
-z^{2} \partial_{z}^{2} v(z)+z\left(3+2 \lambda^{2} z^{2}\right) \partial_{z} v(z)+m_{X}^{2}(z) R^{2} v(z)=0 \\
-\partial_{z}^{2} S_{n}(z)+\left(\frac{3}{z}+2 \lambda^{2} z\right) \partial_{z} S_{n}(z)+\frac{m_{X}^{2}(z) R^{2}}{z^{2}} S_{n}(z)=M_{S}^{2} S_{n}(z) \\
-\partial_{z}^{2} V_{n}(z)+\left(\frac{1}{z}+2 \lambda^{2} z\right) \partial_{z} V_{n}(z)=M_{V}^{2} V_{n}(z) \\
-\partial_{z}^{2} A_{n}(z)+\left(\frac{1}{z}+2 \lambda^{2} z\right) \partial_{z} A_{n}(z)+\frac{R^{2} g_{5}^{2} v^{2}(z)}{z^{2}} A_{n}(z)=M_{A}^{2} A_{n}(z)
\end{gathered}
$$

Before discussing the phenomenology of this model, it is necessary to know the precise form of $m_{X}^{2}(z)$, something we will now consider.

\section{OBTAINING AN EXPRESSION FOR $m_{X}^{2}(z)$}

The mass for scalar modes in the bulk, $m_{X}^{2}(z)$, is obtained starting from (4), although knowing first the func- tion $v(z)$. The behavior of this function is known in two limits.

First we consider the usual limit $z \rightarrow 0$, according to which

$$
v(z \rightarrow 0)=\alpha z+\beta z^{3},
$$

where the $\alpha$ and $\beta$ coefficient are associated with the quark mass and chiral condensate respectively.

The other limit in which we know the behavior of $v(z)$ 

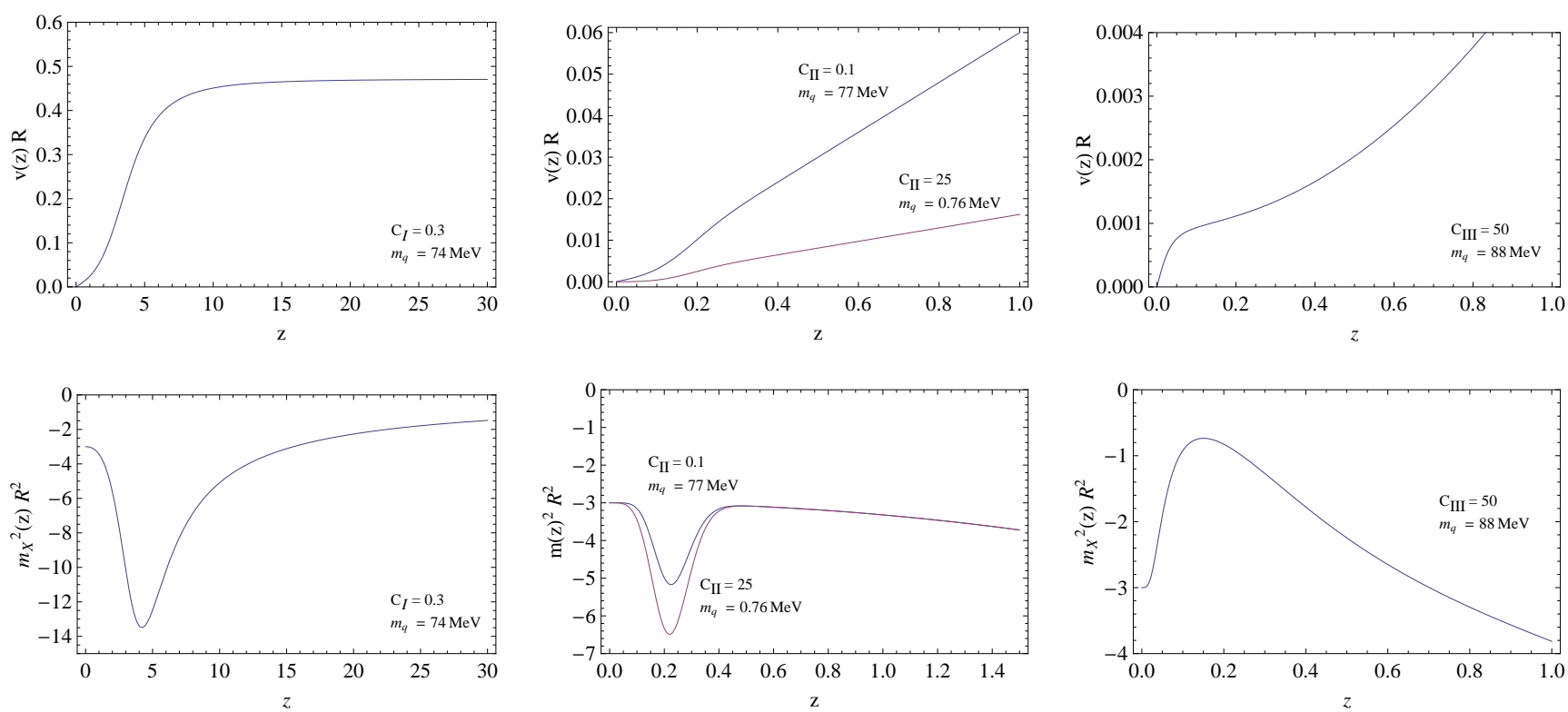

FIG. 1: The upper graph shows $v(z) R$, while in the lower graph the scalar mode masses as functions of $\mathrm{z}$ are given. In the left column, we put plots associated to model I, the middle column is model II, and the right column is model III . All plots have been obtained with $\lambda=0.4 \mathrm{GeV}$, and $c_{I}, c_{I I}$ and $c_{I I I}$ values are shown in each plot.

is when $z \rightarrow \infty$, and therefore we require that (7) gives a Regge-like in this limit. In order to expose this clearly, we change (77) using

$$
A_{n}(z)=\exp \left(\frac{1}{2} \int\left(\frac{1}{z}+2 \lambda^{2} z\right) d z\right) a_{n}(z)
$$

This transformation converts our equation into a Schrödinger like equation, with a potential

$$
V_{A}(z)=\frac{3}{4 z^{2}}+\lambda^{4} z^{2}+\frac{R^{2} g_{5}^{2} v^{2}(z)}{z^{2}} .
$$

As is well known, soft wall models must reproduce spectra with Regge behavior when $z \rightarrow \infty$, so the potential in this limit must look like

$$
V(z)=a+b z^{2}+\frac{c}{z^{2}},
$$

and therefore $v(z \rightarrow \infty)$ can be: constant, linear or quadratic in $\mathrm{z}$.

Knowing the behavior of $v(z)$ both for $z \rightarrow 0$ as in $z \rightarrow \infty$, we choose an ansatz capable to reproduce both limits for each possibility,

$$
\begin{gathered}
v_{I}(z)=\frac{c_{I}}{R} \arctan \left(A z+B z^{3}\right), \quad(\text { Model } I) \\
v_{I I}(z)=\frac{z}{R}\left(A+B \tanh \left(c_{I I} z^{2}\right)\right), \quad(\text { Model } I I) \\
v_{I I I}(z)=\frac{A z+B z^{3}}{R \sqrt{1+c_{I I}^{2} z^{2}}} . \quad(\text { Model III })
\end{gathered}
$$

Using these forms for $v(z)$ in (44) allows us to get an expression for $m_{X}^{2}(z)$. Both $v(z)$ and $m_{X}(z)$ are shown in Fig. 1 considering only parameters that give us good mesonic spectra. The parameters A and B are related to the quark mass and chiral condensate, and its values are fixed according to the discussion in the next section and $c_{I}, c_{I I}$ and $c_{I I I}$ are arbitrary.

\section{PARAMETER SETTING}

The first parameter that we fix is $\lambda$, using data from the spectrum. Specifically we consider a specific value for the Regge slope, which in this kind of models with quadratic dilaton is $4 \lambda^{2}$. In [6] a Regge slope is fixed through radial excitations with $n \geq 3$, but in our case, since the model has Regge behavior in the vector meson sector, we use a value fixed by the lightest vector meson, so finally we choose $\lambda=0.400 \mathrm{GeV}$ that allows us to obtain correct value masses for vector mesons.

The remaining parameters can be fixed using (12), (13) and (14), the expression chosen to describe the vev, for model I

$$
\begin{gathered}
v(z \rightarrow 0)=\frac{\Omega}{R} A z+\frac{\Omega}{R}\left(-\frac{A^{3}}{3}+B\right) z^{3}+O\left(z^{5}\right), \\
v(z \rightarrow \infty)=\frac{\Omega \pi}{2 R}+O\left(z^{-3}\right) .
\end{gathered}
$$

Comparing (15) with the value established in the AdS 
TABLE II: Scalar meson spectra. All masses are in MeV.

\begin{tabular}{c|c|c|c|c|c|c}
\hline \hline $\mathrm{n}$ & $f_{0}(\operatorname{Exp})$ & $f_{0}$ & $f_{0}$ & $f_{0}$ & $f_{0}$ & $f_{0}($ Ref.[6]) \\
& & $m_{q}=74$ & $m_{q}=77$ & $m_{q}=0.8$ & $m_{q}=88$ & \\
& & $c_{I}=0.3$ & $c_{I I}=0.1$ & $c_{I I}=25$ & $c_{I I I}=50$ & \\
\hline 0 & $550_{-150}^{+250}$ & 552 & 583 & 485 & 486 & 799 \\
1 & $980 \pm 10$ & 1089 & 1075 & 903 & 951 & 1184 \\
2 & $1350 \pm 150$ & 1390 & 1350 & 1208 & 1251 & 1466 \\
3 & $1505 \pm 6$ & 1621 & 1574 & 1451 & 1490 & 1699 \\
4 & $1724 \pm 7$ & 1816 & 1769 & 1659 & 1695 & 1903 \\
5 & $1992 \pm 16$ & 1991 & 1943 & 1844 & 1877 & 2087 \\
6 & $2103 \pm 8$ & 2149 & 2103 & 2012 & 2042 & 2257 \\
7 & $2314 \pm 25$ & 2296 & 2251 & 2166 & 2195 & 2414 \\
\hline \hline
\end{tabular}

/ CFT dictionary, with the notation used in [6]

$$
v(z \rightarrow 0)=\frac{m_{q} \zeta}{R} z+\frac{\sigma}{R \zeta} z^{3},
$$

The parameter $\zeta$ was introduced in [10] to get the right normalization, and his value is $\zeta=\sqrt{3} /(2 \pi)$. With this $\mathrm{A}$ and $\mathrm{B}$ parameters are given by

$$
A=\frac{\sqrt{3}}{2 \pi c_{I}} m_{q}
$$

and

$$
B=\frac{2 \pi}{\sqrt{3} c_{I}} \sigma+\frac{\sqrt{3}}{8 \pi^{3} c_{I}^{3}} m_{q}^{3},
$$

where $m_{q}$ is the quark mass and $\sigma$ is the chiral condensate.

In a similar way, for Model II we get

$$
A=\frac{\sqrt{3}}{2 \pi c_{I I}} m_{q}
$$

and

$$
B=\frac{2 \pi}{\sqrt{3} c_{I I}} \sigma
$$

and finally by for Model III we get

$$
A=\frac{\sqrt{3}}{2 \pi} m_{q}
$$

and

$$
B=\frac{9 \sqrt{3}}{4 \pi} m_{q}+\frac{2 \pi}{\sqrt{3}} \sigma,
$$

In order to finish the model description, it is necessary to specify the values for $m_{q}$ and $\sigma$, which are related by the GOR $m_{\pi}^{2} f_{\pi}^{2}=2 m_{q} \sigma$, and therefore we need to fix only one of them. In this case we use $m_{\pi}=140 \mathrm{MeV}$ and $f_{\pi}=92 \mathrm{MeV}$, and we fix the quark mass using

$$
f_{\pi}^{2}=-\left.\frac{1}{g_{5}^{2}} \lim _{\epsilon \rightarrow 0} \frac{\partial_{z} A_{0}(0, z)}{z}\right|_{z=\epsilon}
$$

where $A_{0}(0, z)$ is solution of (7), with $M_{A}^{2}=0$, and the boundary conditions used are $A_{0}(0,0)=1$ and $\partial_{z} A_{0}(0, z \rightarrow \infty)=0$.

As can be observed in (77), the $A_{0}(0, z)$ equation as a term that depends on $m_{q}$, so using (24) we get $f_{\pi}\left(m_{q}\right)$. In general we obtain two possible quark masses in each model, one mass can be considered as a current mass, and only Model II allows us to obtain good mesonic spectra.

\section{MESONIC SPECTRUM}

Having fixed the parameters of the model, we can calculate masses for some mesons, which correspond to eigenvalues in the equations (5), (6) and (7). In this set of equations, only (6) can be solved analytically. For this reason we prefer to change all equations into Schrödinger like ones, and later solve numerically (5) and (7) using a MATHEMATICA code called SCHROEDINGER.nb [21], which was adapted to our potentials.

\section{A. Scalar mesons}

Using the following transformation

$$
S_{n}(z)=\exp \left(\frac{1}{2} \int\left(\frac{3}{z}+2 \lambda^{2} z\right) d z\right) s_{n}(z)
$$

equation (5) is converted in a Schrödinger like equation, with potential given by

$$
V_{S}(z)=2 \lambda^{2}+\lambda^{4} z^{2}+\frac{15}{4 z^{2}}+\frac{m_{X}^{2}(z) R^{2}}{z^{2}} .
$$

Since the potential is complicated, we must solve numerically our Schrödinger equation. Some results obtained using a MATHEMATICA program appear in Table II. As you can see, all models reproduce good spectra, but in general using a higher quark mass, but only Model II offer this possibility with a reasonable quark mass. 


\section{B. Vector mesons}

Notice that in this case equation (6) does not have a dependence on c parameter.

In this case (6) is converted in a Schrödinger equation through

$$
V_{n}(z)=\exp \left(\frac{1}{2} \int\left(\frac{1}{z}+2 \lambda^{2} z\right) d z\right) v_{n}(z)
$$

and the potential is

$$
V_{V}(z)=\frac{3}{4 z^{2}}+\lambda^{4} z^{2}
$$

For this potential it is possible to get an exact spectrum, which is

$$
M_{V}^{2}=4 \lambda^{2}(n+1)
$$

Some examples of masses for this case are shown in Table III, where as you can see the results are satisfactory in general.

TABLE III: Vector mesons spectra in $\mathrm{MeV}$.

\begin{tabular}{c|c|c|c}
\hline \hline $\mathrm{n}$ & $\rho($ Exp $)$ & $\rho($ Model $)$ & $\rho($ Ref.[6] $)$ \\
\hline 0 & $775.5 \pm 1$ & 800 & 475 \\
1 & $1282 \pm 37$ & 1131 & 1129 \\
2 & $1465 \pm 25$ & 1386 & 1529 \\
3 & $1720 \pm 20$ & 1600 & 1674 \\
4 & $1909 \pm 30$ & 1789 & 1884 \\
5 & $2149 \pm 17$ & 1960 & 2072 \\
6 & $2265 \pm 40$ & 2117 & 2243 \\
\hline \hline
\end{tabular}

\section{Axial vector mesons}

As was pointed out in section III, we can transform (7) into a Schrödinger equation using

$$
A_{n}(z)=\exp \left(\frac{1}{2} \int\left(\frac{1}{z}+2 \lambda^{2} z\right) d z\right) a_{n}(z)
$$

and with this, the potential obtained is

$$
V_{A}(z)=\frac{3}{4 z^{2}}+\lambda^{4} z^{2}+\frac{R^{2} g_{5}^{2} v^{2}(z)}{z^{2}} .
$$

In Table IV we show some masses for axial vector mesons.

\section{CONCLUSIONS}

The possibility of incorporating chiral symmetry breaking in soft wall models, introducing a dependence on the holographical coordinate in the mass for models propagating inside the bulk, was studied. This idea could be considered as a complement to other mechanisms that try to solve this problem introducing changes in the dilaton field, changes in the metric, or introducing a cubic or quartic term for scalars in the action [6 6 [

The model considered here considers a usual quadratic dilaton and a AdS metric, and three expressions for $v(z)$ with different limits when $z \rightarrow \infty$. Starting with this it is possible to obtain a mass expression for scalar modes in the bulk. The parameters are fixed using a Gell-MannOakes-Renner relationship and the pion decay constant. An important point to be considered is that for certain choice of parameters the mass of the lightest scalar meson is less than the pion mass, contradicting a wellestablished QCD result. This can be resolved in some cases, at the expense of considering rather large quark masses in models I and III. Model II offers the possibility to built a model that reproduces mesonic spectra with a current quark mass, so it is better than the other two.

We have presented a model that considers AdS modes with variable mass in the bulk, which certainly requires improvements. Extensions of the model should consider a mixture of ingredients, such as non quadratic dilatons and / or asymptotically AdS metrics. Efforts in this direction have already been initiated, and one of the objectives of this paper is to point that a variable mass in the bulk can be viewed as a complement to them.

In this paper the variable mass in the bulk is associated with AdS modes related to operators with anomalous dimensions. In this way a an important property of QCD was considered, which with the exception of a few works, is not taken into account when building models of the AdS / QCD type.

One aspect that is beyond the scope of this work, but it deserves a mention and further study, is related to the running quark mass and the chiral condensate. In this work, the introduction of a z-dependent mass in the bulk is motivated by considering the anomalous dimension for $q \bar{q}$, which should result in the running of the chiral condensate and the mass of the quarks. The interpretation of the mass of the quarks and the chiral condensate is in the limit where $z \rightarrow 0$, so the dependence on $z$ of such quantities is not considered in this model.

\section{Acknowledgments}

We would like to thank Ph.D Franz Schöberl, who provided us the MATHEMATICA program that was used with a small change in this work. Work supported by Fondecyt (Chile) under Grants No. 3100028 and 1100287. 
TABLE IV: Axial vector mesons spectra. All masses are in $\mathrm{MeV}$.

\begin{tabular}{c|c|c|c|c|c|c}
\hline \hline $\mathrm{n}$ & $a_{1}(\operatorname{Exp})$ & $a_{1}$ & $a_{1}$ & $a_{1}$ & $a_{1}$ & $a_{1}($ Ref.[6]) \\
& & $m_{q}=74$ & $m_{q}=77$ & $m_{q}=0.8$ & $m_{q}=88$ & \\
& & $c_{I}=0.3$ & $c_{I I}=0.1$ & $c_{I I}=25$ & $c_{I I I}=50$ & \\
\hline 0 & $1230 \pm 40$ & 867 & 1788 & 811 & 799 & 1185 \\
1 & $1647 \pm 22$ & 1186 & 1959 & 1133 & 1131 & 1591 \\
2 & $1930_{-70}^{+39}$ & 1427 & 2116 & 1384 & 1386 & 1900 \\
3 & $2096 \pm 122$ & 1633 & 2262 & 1601 & 1600 & 2101 \\
4 & $2270_{-40}^{+55}$ & 1816 & 2399 & 1789 & 1789 & 2279 \\
\hline \hline
\end{tabular}

[1] J. Erlich, E. Katz, D. T. Son and M. A. Stephanov, Phys. Rev. Lett. 95, 261602 (2005) arXiv:hep-ph/0501128.

[2] L. Da Rold and A. Pomarol, Nucl. Phys. B 721, 79 (2005) arXiv:hep-ph/0501218.

[3] A. Karch, E. Katz, D. T. Son and M. A. Stephanov, Phys. Rev. D 74, 015005 (2006) arXiv:hep-ph/0602229.

[4] P. Colangelo, F. De Fazio, F. Giannuzzi, F. Jugeau and S. Nicotri, Phys. Rev. D 78, 055009 (2008) arXiv:0807.1054 [hep-ph]].

[5] F. Zuo, arXiv:0909.4240 [hep-ph].

[6] T. Gherghetta, J. I. Kapusta and T. M. Kelley, Phys. Rev. D 79, 076003 (2009) arXiv:0902.1998 [hep-ph]].

[7] H. J. Kwee and R. F. Lebed, Phys. Rev. D 77, 115007 (2008) arXiv:0712.1811 [hep-ph]].

[8] Y. Q. Sui, Y. L. Wu, Z. F. Xie and Y. B. Yang, Phys. Rev. D 81, 014024 (2010) arXiv:0909.3887 [hep-ph]].

[9] P. Zhang, arXiv:1003.0558 [hep-ph].

[10] A. Cherman, T. D. Cohen and E. S. Werbos, Phys. Rev. C 79, 045203 (2009) arXiv:0804.1096 [hep-ph]].

[11] H. Forkel and E. Klempt, Phys. Lett. B 679, 77 (2009)
arXiv:0810.2959 [hep-ph]].

[12] H. Forkel, Phys. Lett. B 694, 252 (2010) arXiv:1007.4341 [hep-ph]].

[13] A. Vega and I. Schmidt, Phys. Rev. D 79, 055003 (2009) arXiv:0811.4638 [hep-ph]].

[14] I. Kirsch, JHEP $0609, \quad 052 \quad$ (2006) arXiv:hep-th/0607205.

[15] H. Forkel, M. Beyer and T. Frederico, JHEP 0707, 077 (2007) arXiv:0705.1857 [hep-ph]].

[16] H. Forkel, M. Beyer and T. Frederico, Int. J. Mod. Phys. E 16, 2794 (2007).

[17] W. de Paula and T. Frederico, arXiv:0908.4282 [hep-ph].

[18] G. F. de Teramond and S. J. Brodsky, Phys. Rev. Lett. 102, 081601 (2009) arXiv:0809.4899 [hep-ph]].

[19] D. Weingarten, Phys. Rev. Lett. 51, 1830 (1983).

[20] E. Witten, Phys. Rev. Lett. 51, 2351 (1983).

[21] W. Lucha and F. F. Schoberl, Int. J. Mod. Phys. C 10, 607 (1999) arXiv:hep-ph/9811453. 